\title{
Spontaneous magnetization of the vacuum and the strength of the magnetic field in the hot Universe
}

\author{
E. Elizalde ${ }^{a *}$ and V. Skalozub ${ }^{b \dagger}$ \\ ${ }^{a}$ Institute for Space Science, ICE-CSIC and IEEC \\ Campus UAB, 08193 Bellaterra, Barcelona, Spain \\ ${ }^{b}$ Dnipropetrovsk National University, 49010 Dnipropetrovsk, Ukraine
}

November 8, 2018

\begin{abstract}
Intergalactic magnetic fields are assumed to have been spontaneously generated at the reheating stage of the early Universe, due to vacuum polarization of non-Abelian gauge fields at high temperature. The fact that the screening mass of this type of fields has zero value was discovered recently. A procedure to estimate their field strengths, $B(T)$, at different temperatures is here developed, and the value $B\left(T_{e w}\right) \sim 10^{14} G$ at the electroweak phase transition temperature, is derived by taking into consideration the present value of the intergalactic magnetic field strength, $B_{0} \sim 10^{-15} \mathrm{G}$, coherent on the $\sim 1 \mathrm{Mpc}$ scale. As a particular case, the standard model is considered and the field scale at high temperature is estimated in this case. Model dependent properties of the phenomena under investigation are briefly discussed, too.
\end{abstract}

\section{Introduction}

The recent experimental discovery of intergalactic magnetic fields having a field strength of the order $B \sim 10^{-15} G$ is one of the most interesting events of modern cosmology [1, 2]. In Ref. 3] a model-independent, 95\% CL interval $1 \times 10^{-17} G \leq B \leq 3 \times 10^{-14} G$ was determined, and the femtoGauss values are actual field strengths in extragalactic space. This means, to start, that magnetic

*E-mail: elizalde@ieec.uab.es, elizalde@math.mit.edu

†E-mail: skalozubv@daad-alumni.de 
fields are actually present everywhere in the Universe and influence various processes. Secondly, this renders most likely the cosmological origin of primordial magnetic fields. From the theoretical viewpoint, this discovery restricts in an essential way the possible processes which may result in the creation of fields in the hot Universe [2]. As a consequence, the search for mechanisms of field generation has intensified. The most obvious candidates are primordial fluctuations, but there are other (for a review, see [4]-[6]). The challenge is to produce coherent magnetic fields on very long scales in an almost empty intergalactic space. In this paper we will discuss some mechanism based on non-Abelian magnetic fields.

As was shown recently, spontaneous vacuum magnetization appears in nonAbelian gauge theories at high temperature. This was found by analytic methods in [7]-[10] and was confirmed by means of lattice simulations in [11]. The basic idea rests on the known fact that spontaneous vacuum magnetization is the consequence of the spectrum of a color charged gluon,

$$
p_{0}^{2}=p_{\|}^{2}+(2 n+1) g B \quad(n=-1,0,1, \ldots),
$$

in a homogeneous magnetic background, $B$, described by the potential

$$
A_{\mu}^{a}=B x_{2} \delta_{\mu 3} \delta^{a 3}
$$

where $a$ is the weak isotopic index, and $p_{\|}$a momentum component along the field direction. Here, a tachyon mode is present in the ground state $(n=-1)$. In fact, one observes that $p_{0}^{2}<0$, resulting from the interaction of the magnetic moment of the spin-1 charged particles with the magnetic field. This phenomenon was first discovered by Savvidy [12] at zero temperature, $T=0$, and got known as the Savvidy vacuum. However, at zero and low temperature, this state is not stable. It decays under emission of gluons until the magnetic field $B$ disappears. This picture changes with increasing temperature, where a stabilization sets in. The stabilization is due to vacuum polarization and depends on two dynamical parameters appearing for $T \neq 0$. These is a magnetic mass of the color charged gluon, $m_{\text {magn. }}$, and an $A_{0}$-condensate, which is proportional to the Polyakov loop [13. This field configuration is stable, its energy being below the perturbative one, and its minimum is reached for the field being of order $g B \sim g^{4} T^{2} / \log T$. This phenomenon is common to different $S U(N)$ gauge fields, which can be used to extend the standard $S U(2) \times U(1))_{e w} \times S U(3)_{c}$ model of elementary particles.

An important property of such temperature-dependent magnetic fields is the vanishing of their magnetic mass, $m_{\text {magn. }}=0$. This was found both in one-loop analytic calculations [14] and in lattice simulations [15]. The mass parameter describes the inverse spatial scales of the transverse field components, similarly to the Debye mass $m_{D}$, related to the inverse space scale for the electric (Coulomb) component. The absence of the screening mass means that the spontaneously generated Abelian chromomagnetic fields are long range at high temperature, as is common for the $U(1)$ magnetic field. Hence, it is reasonable to believe that, 
at each stage of the evolution of the hot Universe, spontaneously created, strong, long-range magnetic fields of different types have been present. Since they are constant fields, their scales are coinciding with the horizon scale at each particular temperature. These fields may have critically influenced various processes and phase transitions.

The idea of the present investigation is to relate the generation of intergalactic magnetic fields with the reheating epoch which immediately followed the inflationary stage of the universe evolution. At this epoch the temperature was the same in all volume of the expanded space (as it follows from the spectra of relic photons), and therefore, the field strength was also the same. Moreover, since both inflation and the vacuum polarization are causal processes, the coherence of the field at very large scales can be ensured. On the other hand, the creation of strong temperature dependent fields at this stage completely washed out the remnants of the fields generated at the inflation epoch.

The dependence on the temperature of these fields differs from that of the usual $U(1)$ magnetic fields. Recall that, in the latter case, as is commonly assumed the magnetic (hypermagnetic, in fact) field, created by some specific mechanism, is implemented in a hot plasma and evolves according to the law $B \sim T^{2}$, which is a consequence of magnetic flux conservation (see, for instance, [6]). This scenario is actually considered, in magnetic hydrodynamics, when the evolution of large scale magnetic fields is investigated. The temperature dependence of a field of this type is ordinarily related with the high conductivity of the plasma. Thus, it is just a consequence of the classical theory of matter. However, the classical theory is not always sufficient for describing the restored phase in models with spontaneous symmetry breaking. In this phase, quantum effects are essential. One of them is vacuum polarization, which results in spontaneous vacuum magnetization. Just this phenomenon regulates the dependence of the field strength on the temperature, and the magnetic flux is not conserved. Instead, a specific flux value is generated at each temperature. This fact has to be taken into consideration when the cooling pattern of the hot plasma is investigated at temperatures over that of the electroweak phase transition (EWPT), $T \geq T_{e w} \sim 100 \mathrm{GeV}$. This also concerns the $S U(2)_{e w}$ component of the electromagnetic field. The classical theory is not able to account for it consistently. Thus, classical magnetic hydrodynamics could only start to work after the vacuum magnetization has stopped. As we will show below, this does happen after the EWPT.

It is worth to note that one of the difficult problems of magnetogenesis is to relate the field strengths generated via some given mechanism in the early Universe with those of the present-day fields, what depends on numerous factors and is very model dependent. In magnetic hydrodynamics, the magnetic field evolution was investigated in some detail for stochastic fields presumedly generated at either the electroweak or the confinement phase transitions (see [16, 17], and references therein). It was shown that their fate is strongly dependent on the scales 
and initial conditions for these fields. In particular, magnetic flux conservation could not hold for some special conditions. We make a detailed comparison of these results with those coming from our approach, in what follows. An important point is that, in contrast with commonly investigated stochastic fields, we have here the possibility to deal with the evolution of a special solution of the field equations. Thus, its characteristics and properties are known and can be duely taken into consideration.

In the frameworks of the standard model, we will here estimate the strength of the magnetic field at the temperature of the electroweak $T_{c}^{\mathrm{ew}}$ phase transition, assuming that this field was spontaneously generated by a mechanism as described above. Although such phenomenon is nonperturbative, we carry out an actual calculation in the framework of a consistent effective potential (EP) accounting for the one-loop, $V^{(1)}$, and the daisy (or ring), $V^{\text {ring }}$, diagram contributions. In Sect. 2 we qualitatively describe, in quite more detail, the main aspects of the investigated phenomena. In Sect. 3 the EP of an Abelian constant electromagnetic $B$ field at finite temperature is obtained. It is used, in Sect. 4, to estimate the magnetic field strength at the EWPT temperatures. The scales of the magnetic fields at high temperatures, and their relation with the ones observed in intergalactic space, are investigated in Sect. 5. A discussion of the results obtained, some conclusions, and prospects for further work are provided in the last section.

\section{Qualitative considerations}

In this section we describe, in a qualitative manner, the main aspects of the phenomena at issue. They are all consequences of asymptotic freedom and spontaneous symmetry breaking at finite temperature. Our main assumption is that the intergalactic magnetic field has been spontaneously created at high temperature. We believe, this to be a fairly reasonable idea because, physically, magnetization is the consequence of a large magnetic moment for charged non-Abelian gauge fields (recall the gyromagnetic ratio $\gamma=2$ for $W$-bosons). This property results in the asymptotic freedom of the model in external fields. We discuss the procedure to relate the present value of the intergalactic magnetic field with the one generated in the restored phase.

First, we note that, in non-Abelian gauge theories, magnetic flux conservation does not hold at high temperatures, $T \geq T_{\text {ew }}$, where $T_{\text {ew }}$ is the temperature of EWPT. This is due to spontaneous vacuum magnetization, which depends on the temperature. The vacuum acts as a specific source generating classical fields. Second, the magnetization is strongly dependent on the scalar field condensate present in the vacuum at low temperature. This point was investigated at zero temperature by Goroku [18]. For finite temperature, this is considered in the present paper for the first time. The observation is that, in both cases, the spontaneous vacuum magnetization takes place for small values of the scalar 
field, $\phi \neq 0$, only. For the values of $\phi$ corresponding to any first order phase transition it does not happen. This means that, after the EWPT, the vacuum polarization ceases to generate magnetic fields, and magnetic flux conservation holds. As a result, the familiar dependence on the temperature, $B \sim T^{2}$, is restored (for the fields spontaneously generated before the transition). However, that may be not the case, in general, for stochastic magnetic fields generated just at this transition [16]. One has to distinguish these types of fields, which have different nature and origin.

Another aspect of the problem is the composite structure of the electromagnetic field $A_{\mu}$. The potentials read

$$
\begin{aligned}
& A_{\mu}=\frac{1}{\sqrt{g^{2}+g^{\prime 2}}}\left(g^{\prime} A_{\mu}^{3}+g b_{\mu}\right) \\
& Z_{\mu}=\frac{1}{\sqrt{g^{2}+g^{\prime 2}}}\left(g A_{\mu}^{3}-g^{\prime} b_{\mu}\right)
\end{aligned}
$$

where $Z_{\mu}$ is the $Z$-boson potential, $A_{\mu}^{3}, b_{\mu}$ are the Yang-Mills gauge field third projection in the weak isospin space and the potential of the hypercharge gauge fields, and $g$ and $g^{\prime}$ are $S U(2)$ and $U(1)_{Y}$ couplings, correspondingly. After the electroweak phase transition, the $Z$-boson acquires a mass and the field is screened. Since the hypermagnetic field is not spontaneously generated, only the component $A_{\mu}=g^{\prime} A_{\mu}^{3} / \sqrt{g^{2}+g^{\prime 2}}=\sin \theta_{\mathrm{W}} A_{\mu}^{3}$ is present at high temperature. Here $\theta_{\mathrm{W}}$ is the Weinberg angle, $\tan \theta_{\mathrm{W}}=g^{\prime} / g$. This is the only component responsible for the intergalactic magnetic field at low temperature. In the restored phase, $b_{\mu}=0$, the complete weak-isospin chromomagnetic field $A_{\mu}^{(3)}$ is unscreened. This is because the magnetic mass of this field is zero [15]. Thus, the field is of long range and this provides the coherence length $\lambda_{B}(T)$ to be sufficiently large. In fact, because the field is a constant, it has to cover all the horizon scale at the given temperature, $\lambda_{B}(T) \sim R_{H(T)}$. This property is very important for our scenario of intergalactic magnetic field generation. Its cosmological consequences will be discussed below. In particular, magnetic fields of different types (color $S U(3)$, and others) can be spontaneously generated at high temperatures.

Here, we continue with the description of the general field behavior related with the EWPT. In the restored phase, a scalar field condensate $\phi=0$, and the constituent of the weak isospin field corresponding to the magnetic one, is given by the expression

$$
B(T)=\sin \theta_{w}(T) B^{(3)}(T),
$$

where $B^{(3)}(T)$ is the strength of the field generated spontaneously. After the phase transition, the scalar condensate is $\phi \neq 0$, and the field is partially screened.

To relate the present value of the intergalactic magnetic field with the field which existed before the EWPT, we take into consideration that, after the phase transition, spontaneous vacuum magnetization does not take place. This property is derived in the next sections. Therefore, for the EWPT temperature $T_{\text {ew }}$, we 
can write

$$
\frac{B\left(T_{e w}\right)}{B_{0}}=\frac{T_{e w}^{2}}{T_{0}^{2}}=\frac{\sin \theta_{w}\left(T_{e w}\right) B^{(3)}\left(T_{e w}\right)}{B_{0}} .
$$

Here, $B_{0}$ is the present value of the intergalactic magnetic field strength $B_{0} \sim$ $10^{-15} G$. The left-hand-side relates the value $B\left(T_{e w}\right)$ with $B_{0}$. The right-handside gives a possibility to express the weak isospin magnetic field in the restored phase through $B_{0}$, knowing the temperature dependence of the Weinberg angle $\theta_{w}(T)$. This relation contains an arbitrary temperature normalization parameter $\tau$. It can be fixed for a given temperature and $B_{0}$. After that, the field strength values at various temperatures can be calculated. In particular, the total weak isospin field strength is given by the sum $\cos \theta_{w}\left(T_{e w}\right) B^{(3)}\left(T_{e w}\right)+B\left(T_{e w}\right)$.

Note also that the relation (5) is a consequence of the assumption that, for the field spontaneously generated at high temperature, the magnetic flux conservation holds, in the early Universe, after the EWPT. This means that the field is "frozen" in the plasma at large scales, and the magnetic turbulence processes do not affect that behavior. Although this is a most simple assumption, it requires a detailed discussion encompassing the results obtained recently in magnetic hydrodynamics (see Refs. [16, 17], and references therein). Details on the magneto-hydrodynamical processes occurring in the early universe can be found in numerous publications (see, for references, the review papers [5, 6]). Here, we will just mention the main points which are important for our future considerations. The "frozen in" conditions are always realized for magnetic fields having the scales larger than the largest turbulence eddies. After a free decay stage of the magnetized plasma evolution, the field can be considered to be nonaffected by turbulence [17]. In connection with these results, it is clear that the magnetic fields generated at the EWPT are not sufficient, as such, to produce long-range correlated fields, and some additional processes must be included. This is because, even a field having the scale of the Hubble radius at temperature $T_{e w} \sim 100 \mathrm{GeV}$, is in fact correlated at the comoving scale $l_{0} \sim 10^{-4} \mathrm{Mpc}$ [17].

But, in the case of a temperature-dependent vacuum polarization, other possibilities exist. To obtain a large-scale correlated intergalactic magnetic field one can take into account that the temperature in the Universe is the same, to high accuracy, and of the order $\delta T \sim 10^{-4}$. This is ensured by the reheating stage coming just after inflation. The temperatures at this stage are estimated to be of the order $10^{16}-10^{12} \mathrm{GeV}$ depending on the specific inflation model [19]. Hence, with high accuracy, the magnetic field strengths $B(T)$ generated due to vacuum polarization are also the same in all regions of the universe. Since they are produced by a causal process, as inflation is, a correlation of the fields at very large scale will result. In this way, coherent magnetic fields on huge scales could be generated. Such kind of fields have much larger scales than any turbulence eddy, therefore, they will not be influenced by turbulent processes. Thus, it is reasonable to assume that these magnetic fields were frozen into plasma after the EWPT. 
An important aspect of the present scenario is that the knowledge of the particular theory, still unknown today, which may prove in future experiments to be the right extension to the standard model, is not that very important for estimating the field strength $B$ at temperatures close to $T_{e w}$. This is so because the new gauge fields of the extended model will remain screened, at the high temperatures corresponding to the spontaneous symmetry breaking of some of the basic symmetries. At very high temperatures, when these symmetries will be restored, the corresponding magnetic fields do will exist. In this way the value of the field strength at the Planck era can be estimated (see Ref. [20], for comparison).

\section{Effective potential at high temperature}

As we noted above, the spontaneous vacuum magnetization and the absence of the magnetic mass for the Abelian magnetic fields are nonperturbative effects to be determined, in particular, in lattice simulations [11, 15]. The main conclusions of these investigations are that a stable magnetized vacuum does exist at high temperature and that the magnetic mass of the created field is zero. Concerning the actual value of the field strength, is is close to the one calculated within the consistent effective potential which takes into account one-loop plus daisy diagrams. Thus, in the present investigation we restrict ourselves to such approximation. This is mainly with the purpose to be able to use analytic calculations in order to properly interpret the results.

The complete EP for the standard model is given in the review [21]. In the present investigation we are interested in two limits,

1. weak magnetic field and large scalar field condensate, $h=e B / M_{W}^{2}<\phi^{2}$, $\phi=\phi_{c} / \phi_{0}, \beta=1 / T$

2. the case of the restored symmetry, $\phi=0, g B \neq 0, T \neq 0$.

For the first case, we show the absence of spontaneous vacuum magnetization at finite temperature. For the second, we estimate the field strength at high temperature. Here $M_{W}$ is the $W$-boson mass at zero temperature, $\phi_{c}$ a scalar field condensate, and $\phi_{0}$ its value at zero temperature.

To demonstrate the first property we consider the one-loop contribution of $W$-bosons (see also Eq. (27) of Ref. [9]),

$$
\begin{aligned}
V_{W}^{(1)}(T, h, \phi)= & \frac{h}{\pi^{2} \beta^{2}} \sum_{n=1}^{\infty}\left[\frac{\beta \sqrt{\phi^{2}-h}}{n} K_{1}\left(n \beta \sqrt{\phi^{2}-h}\right)\right. \\
& \left.-\frac{\beta \sqrt{\phi^{2}+h}}{n} K_{1}\left(n \beta \sqrt{\phi^{2}+h}\right)\right] .
\end{aligned}
$$

Here $n$ labels discrete energy values and $K_{1}(z)$ is the MacDonald function. 
The main goal of our investigation is the restored phase of the standard model. So, we obtain the high temperature contribution of the complete effective potential relevant for this case using the results in Ref. [21]. First, we write down the one-loop $W$-boson contribution as the sum of the pure Yang-Mills weak-isospin $\operatorname{part}\left(\tilde{B} \equiv B^{(3)}\right)$,

$$
\begin{aligned}
V_{W}^{(1)}(\tilde{B}, T) & =\frac{\tilde{B}^{2}}{2}+\frac{11}{48} \frac{g^{2}}{\pi^{2}} \tilde{B}^{2} \log \frac{T^{2}}{\tau^{2}}-\frac{1}{3} \frac{(g \tilde{B})^{3 / 2} T}{\pi} \\
& -i \frac{(g \tilde{B})^{3 / 2} T}{2 \pi}+O\left(g^{2} \tilde{B}^{2}\right)
\end{aligned}
$$

where $\tau$ is a temperature normalization point, and the charged scalars [9],

$$
V_{\mathrm{sc}}^{(1)}(\tilde{B}, T)=-\frac{1}{96} \frac{g^{2}}{\pi^{2}} \tilde{B}^{2} \log \frac{T^{2}}{\tau^{2}}+\frac{1}{12} \frac{(g \tilde{B})^{3 / 2} T}{\pi}+O\left(g^{2} \tilde{B}^{2}\right),
$$

describing the contribution of longitudinal vector components. The first term in Eq. (7) is the tree-level energy of the field. This representation is convenient for the case of extended models including other gauge and scalar fields. Depending on the specific case, one can take into consideration the parts (7) and (8), correspondingly. In the standard model, the contribution of Eq. (8) has to be taken with a factor 2 , due to the two charged scalar fields entering the scalar doublet of the model. In the case of the Two-Higgs-Doublet standard model, this factor must be 4 , etc. The imaginary part is generated because of the unstable mode in the spectrum (1). It is canceled by the term appearing in the contribution of the daisy diagrams for the unstable mode [10],

$$
V_{\text {unstable }}=\frac{g \tilde{B} T}{2 \pi}[\Pi(\tilde{B}, T, n=-1)-g \tilde{B}]^{1 / 2}+i \frac{(g \tilde{B})^{3 / 2} T}{2 \pi} .
$$

Here $\Pi(\tilde{B}, T, n=-1)$ is the mean value for the charged gluon polarization tensor taken in the ground state $n=-1$ of the spectrum (11). If this value is sufficiently large, spectrum stabilization due to radiation correction takes place. This possibility formally follows from the temperature and field dependence of the polarization tensor in the high temperature limit $T \rightarrow \infty[22]: \Pi(\tilde{B}, T, n=$ $-1)=c g^{2} T \sqrt{g \tilde{B}}$, where $c>0$ is a constant which must be calculated explicitly. At high temperature the first term can be larger then $g \tilde{B}$. From Eqs. (7) and (9) it follows that the imaginary part is canceled. Hence, we see that having accounted for rings leads to vacuum stabilization even if $\Pi(\tilde{B}, T, n=-1)$ is smaller then $g \tilde{B}$. Really, in the latter case, the imaginary part will be smaller than in Eq. (17). The high temperature limit of the fermion contribution looks as

$$
V_{\text {fermion }}=-\frac{\alpha}{\pi} \sum_{f} \frac{1}{6} q_{f}^{2} \tilde{B}^{2} \log \frac{T}{\tau}
$$


where the sum is extended to all leptons and quarks, and $q_{f}$ is the fermion electric charge in positron units. Hence, it follows that in the restored phase all the fermions give the same contribution.

Let us now present the EP for ring diagrams describing the long range correlation corrections at finite temperature [23, 24],

$$
\begin{aligned}
V_{\text {ring }} & =\frac{1}{24 \beta^{2}} \Pi_{00}(0)-\frac{1}{12 \pi \beta} \operatorname{Tr}\left[\Pi_{00}(0)\right]^{3 / 2} \\
& +\frac{\left(\Pi_{00}(0)\right)^{2}}{32 \pi^{2}}\left[\log \frac{4 \pi}{\beta\left(\Pi_{00}(0)\right)^{1 / 2}}+\frac{3}{4}-\gamma\right],
\end{aligned}
$$

where the trace means summation over all the contributing states, $\Pi_{00}=\Pi_{\phi}(k=$ $0, T, B)$ for the Higgs particle; $m_{D}^{2}=\Pi_{00}=\Pi_{00}(k=0, T, B)$ are the zerozero components of the polarization functions of gauge fields in the magnetic field taken at zero momenta, called the Debye mass squared, and $\gamma$ is Euler's gamma. These terms are of order $\sim g^{3}\left(\lambda^{3 / 2}\right)$ in the coupling constants. The detailed calculation of these functions is given in Ref. [21]. We give the results for completeness,

$$
\begin{aligned}
\Pi_{\phi}(0) & =\frac{1}{24 \beta^{2}}\left(6 \lambda+\frac{6 e^{2}}{\sin ^{2}\left(2 \theta_{w}\right)}+\frac{3 e^{3}}{\sin ^{2} \theta_{w}}\right) \\
& +\frac{2 \alpha}{\pi} \sum_{f}\left[\frac{\pi^{2} K_{f}}{3 \beta^{2}}-\left|q_{f} B\right| K_{f}\right] \\
& +\frac{(e B)^{1 / 2}}{8 \pi \sin ^{2} \theta_{w} \beta} e^{2}\left(3 \sqrt{2} \zeta\left(-\frac{1}{2}, \frac{1}{2}\right)\right) .
\end{aligned}
$$

Here $K_{f}=\frac{m_{f}^{2}}{M_{w}^{2}}=\frac{G_{Y u k a w a}^{2}}{g^{2}}$ and $\lambda$ is the scalar field coupling. The terms $\sim T^{2}$ yield standard contributions to the temperature mass squared coming from the boson and fermion sectors. The $B$-dependent terms are negative (note the value of $\left.3 \sqrt{2} \zeta\left(-\frac{1}{2}, \frac{1}{2}\right)=-0.39\right)$. They decrease the value of the screening mass at high temperature. The Debye masses squared for the photons, $Z$-bosons, and neutral current contributions are, respectively,

$$
\begin{aligned}
& m_{D, \gamma}^{2}=g^{2} \sin ^{2} \theta_{\mathrm{W}}\left[\frac{1}{3 \beta^{2}}+O\left(e B \beta^{2}\right)\right], \\
& m_{D, Z}^{2}=g^{2}\left(\tan ^{2} \theta_{\mathrm{W}}+\frac{1}{4 \cos ^{2} \theta_{\mathrm{W}}}\right)\left[\frac{1}{3 \beta^{2}}+O\left(e B \beta^{2}\right)\right], \\
& m_{D, \text { neutr. }}^{2}=\frac{g^{2}}{8 \beta^{2} \cos ^{2} \theta_{\mathrm{W}}}\left(1+4 \sin ^{4} \theta_{\mathrm{W}}\right)+O\left(e B \beta^{2}\right) .
\end{aligned}
$$

As one can see, the dependence on $B$ appears at order $O\left(T^{-2}\right)$.

The $W$-boson contribution to the Debye mass of the photons is

$$
m_{D, W}^{2}=3 g^{2} \sin ^{2} \theta_{\mathrm{W}}\left(\frac{1}{3 \beta^{2}}-\frac{\left(g \sin \theta_{\mathrm{W}} B\right)^{1 / 2}}{2 \pi \beta}\right) .
$$


An interesting feature of this expression is the negative sign of the next-to-leading terms which dependen on the field strength. Finally, we give the contribution of the high temperature part in Eq. (9) $\Pi(\tilde{B}, T, n=-1)$ [21],

$$
\Pi(\tilde{B}, T, n=-1)=\alpha \frac{\left(g \sin \theta_{\mathrm{W}} B\right)^{1 / 2}}{\beta}(12.33+4 i) .
$$

This expression was calculated from the one-loop $W$-boson polarization tensor in the external field at high temperature. It contains the imaginary part which comes from the unstable mode in the spectrum (1). Its value is small as compared to the real one. It is of the order of the usual damping constants in plasma at high temperature. It will be thus ignored in actual calculations in what follows. In fact, this part must be calculated in a more consistent scheme which starts with a regularized stable spectrum. On the other hand, as we noted above, the stability problem is a non-perturbative one. Stabilization can be realized not only through radiation corrections but also by some other mechanisms. For example, due to $A_{0}$ condensation [7] at high temperature. A stable vacuum state was observed in lattice simulations [11, therefore, we believe that this problem has a positive solution. Summing up, we have all necessary ingredients to investigate the problem of interest.

\section{Magnetic field strength at $T_{e w}$}

Let us now show that the spontaneous vacuum magnetization does not take place at finite temperature and for non-small values of the scalar field condensate $\phi \neq 0$. To this end we notice that the magnetization is produced by the gauge field contribution, given in Eq. (6). So, we consider the limit of $g B / T^{2} \ll 1$ and $\phi^{2}>h$. For this case we use the asymptotic expansion of $K_{1}(z)$,

$$
K_{1}(z) \sim \sqrt{\frac{\pi}{2 z}} e^{-z}\left(1+\frac{3}{8 z}-\frac{15}{128 z^{2}}+\ldots\right),
$$

where $z=n \beta\left(\phi^{2} \pm h\right)^{1 / 2}$. Now, we investigate the limit of $\beta \rightarrow \infty, T / \phi \ll 1$, where the leading contribution is given by the first term of the temperature sum in Eq. (6). We can also substitute $\left(\phi^{2} \pm h\right)^{1 / 2}=\phi\left(1 \pm \frac{h}{2 \phi^{2}}\right)$. In this approximation, the sum of the tree level energy and (므) reads

$$
V=\frac{h^{2}}{2}-\frac{h^{2}}{\pi^{3 / 2}} \frac{T^{1 / 2}}{\phi^{1 / 2}}\left(1-\frac{T}{2 \phi}\right) e^{-\phi / T} .
$$

The second term is exponentially small and the stationary equation $\partial V / \partial h=0$ has the trivial solution $h=0$. This estimate can be easily verified in a numerical calculation of the total effective potential. Hence, we conclude that after symmetry breaking the spontaneous vacuum magnetization does not take place, as was the case at zero temperature [18]. 
To estimate the magnetic field strength in the restored phase at the EWPT temperature the total EP deduced in the previous section must be used and the parameters entering Eq. (5) need to be calculated. This can be best done numerically. To explain the procedure, we consider here a part of this potential accounting for the one-loop $W$-boson contributions. The high temperature expansion for the EP coming from charged vector fields is given in Eq. (7). Assuming stability of the vacuum state, we calculate the value of the chromomagnetic weak isospin field spontaneously generated at high temperature from Eqs. (7) and (86):

$$
\tilde{B}(T)=\frac{1}{16} \frac{g^{3}}{\pi^{2}} \frac{T^{2}}{\left(1+\frac{5}{12} \frac{g^{2}}{\pi^{2}} \log \frac{T}{\tau}\right)^{2}} .
$$

This expression (and the complete one accounting for all contributions) gives the field strength at any temperature, $T \geq T_{e w}$. These formulas can be obtained for different types of particles. Before reducing to a specific value for it, we describe how to connect this expression with the intergalactic magnetic field $B_{0}$. We first relate the expression (18) with an electromagnetic field after symmetry breaking, and then take into account the scales of the fields.

Let us introduce the standard parameters and definitions, $\alpha_{\mathrm{w}}=g^{2} /(4 \pi)$, $\alpha=\alpha_{\mathrm{w}} \sin \theta_{\mathrm{W}}^{2}, \alpha_{Y}=\left(g^{\prime}\right)^{2} /(4 \pi)$ and $\tan ^{2} \theta_{\mathrm{W}}(T)=\alpha_{Y}(T) / \alpha_{\mathrm{w}}(T)$, where $\alpha$ is the fine structure constant. To find the temperature dependence of the Weinberg angle, the behavior of the hypercharge coupling $g^{\prime}$ on the temperature has to be computed. From Eq. (8) it follows that this behavior is nontrivial. The logarithmic temperature-dependent term is negative. But, as is well known, in the asymptotically free models this sign must be changed into a positive value, due to the contributions of other fields. This particular value is model dependent. We will not calculate it in the present paper. Instead, for a rough estimate, we substitute the zero-temperature value: $\sin ^{2} \theta_{\mathrm{W}}(T)=\sin ^{2} \theta_{\mathrm{W}}(0)=0.23$.

For the given temperature of the EWPT, $T_{\text {ew }}$, the magnetic field is

$$
B\left(T_{\mathrm{ew}}\right)=B_{0} \frac{T_{\mathrm{ew}}^{2}}{T_{0}^{2}}=\sin \theta_{\mathrm{W}}\left(T_{\mathrm{ew}}\right) \tilde{B}\left(T_{\mathrm{ew}}\right) .
$$

Assuming $T_{\text {ew }}=100 \mathrm{GeV}=10^{11} \mathrm{eV}$ and $T_{0}=2.7 \mathrm{~K}=2.3267 \cdot 10^{-4} \mathrm{eV}$, we obtain

$$
B\left(T_{\text {ew }}\right) \sim 1.8510^{14} G .
$$

This value can be considered as an estimate of the magnetic field strength at the EWPT. Hence, for the value of $X=\log \frac{T_{e w}}{\tau}$, we have the equation

$$
B_{0}=\frac{1}{2} \frac{\alpha^{3 / 2}}{\pi^{1 / 2} \sin ^{2} \theta_{w}} \frac{T_{0}^{2}}{\left(1+\frac{5 \alpha}{3 \pi \sin ^{2} \theta_{w}} X\right)^{2}} .
$$

Since all the values are known, $\log \tau$ can be computed. After that, the field strengths at different higher temperatures can be found. In fact, the main point 
in obtaining these results is the assumption of magnetic flux conservation, as is frozen in a plasma. Information of a particular model is implemented in the factor $\sin \theta_{w}\left(T_{e w}\right)$ in Eq. (19). Needles to say, our estimate is a rough one, because we have ignored the temperature dependence of the Weinberg angle. To guess the value of the parameter $\tau$ we take the field strength $B_{0} \sim 10^{-9} G$, usually used in cosmology (see, e.g., [20]). In this case, from Eq. (21) we obtain $\tau \sim 300 \mathrm{eV}$. For the present-day value $B_{0} \sim 10^{-15} G$ this parameter is much smaller.

To take into account the fermion contribution Eq. (10), we have to substitute the expression $\frac{5}{12} \frac{g^{2}}{\pi^{2}} \log \frac{T}{\tau}$ in Eq. (18) and also in (21), with the value

$$
\left(\frac{5}{3}-\sum_{f} \frac{1}{6} q_{f}^{2}\right) \frac{\alpha_{s}}{\pi} \log \frac{T}{\tau} .
$$

In the above estimate, we have accounted for the one-loop part of the EP of order $\sim g^{2}$ in the coupling constant. The ring diagrams have order $\sim g^{3}$ and give a small numeric correction to this result. As was mentioned before, had we taken into account all the terms listed in the previous section, the results would have not changed essentially.

Let us compare now the value of the field strength (20) with the one calculated directly from the EP for the standard model in Ref. [25]. From Fig. 1 and Tab. 1 of that paper, we find

$$
B^{S M}\left(T_{e w}\right) \sim 10^{20} G,
$$

what is much larger than the value (20) and just corresponds to the value of the present comoving field strength $B_{0} \sim 10^{-9} G$. Note that this value was used in numerous investigations of magnetic fields in the early universe, before the recent discoveries [1]-3]. Let us stress again that the field strength at higher temperatures will depend on the particular model extending the standard one. Spontaneous vacuum magnetization in the minimal supersymmetric standard model has been investigated in Ref. [24], and the field strength generated in this model is smaller as compared to the situation here considered. Also, Pollock [20] has investigated this problem for the case of the Planck era, where magnetic fields of the order $B \sim 10^{52} G$ have been estimated.

\section{Magnetic field scale}

We now discuss in brief the scale of the field generated in the restored phase. This is a key point in relating expressions like Eqs. (18) or (23) with $B_{0}$. In our consideration, the "frozen in" condition was used. Therefore, we are going to discuss its applicability in more detail. Note first that, if one assumes that after the EWPT the constant field $B\left(T_{e w}\right)$ was frozen in the plasma at the Hubble scale, $R_{H}\left(T_{e w}\right)$, then its comoving coherence scale at present will be $\lambda_{B}\left(T_{0}\right)=6 \cdot 10^{-4}$ pc [17]. This is much smaller than is needed. 
We propose two, in fact related, ways to overcome such difficulty. The first is to take into consideration the reheating stage of the universe evolution. According to the concepts of modern cosmology [19], this stage has existed just after inflation and is related with the latter causal stage. Just due to causality, the temperature in the universe after this stage is the same, in all domains of space, which could even be uncorrelated in later moments of time. Hence, at a given high temperature, $T$, the magnetic field generated due to vacuum polarization has the same strength $B(T)$ everywhere in the universe. Formally, they could have different directions, in either external or internal spaces, although this point requires additional consideration. Different kind of chromomagnetic fields, of the type as in Eq. (2), can be spontaneously generated. Their nature depends on the particular model considered and is therefore unknown, as of now. But this is not essential for our consideration, here. The magnetic fields coherent on huge scales are expected to have been present in the early Universe. The origin of this coherence is ensured by the properties of the solution to the field equations (Eq. (2)) and by causality at the inflationary epoch. The scales of the coherent field domains could be estimated on grounds of the gauge invariance. This idea, due to Feynman, was put in force in gluodynamics with the goal to determine possible magnetic vacuum structures [26]. Namely, to find a gauge invariant vacuum, on the basis of gauge non-invariant solutions (such as Eq. (2)), one can consider a domain structure ensuring gauge invariance when a corresponding boundary is going around. This point requires further investigation.

Most of the fields generated in the early Universe decouple and are screened at some energy (temperature) scales, when the corresponding scalar condensates have broken the background symmetries. So, the only unbroken symmetry at the EWPT remains the $S U(2)_{e w} \times U(1)$ one. After the EWPT, when spontaneous magnetization stops, this field cannot be included in turbulent processes generated by the transition. This is because the scale of the field, Eq. (2), is already much larger than any largest eddy of turbulence. As is usually believed, the size of a typical eddy is estimated as the inverse mass of the particles appearing after the transition [27]. Thus, the field evolves in accordance with the metric expansion and is implemented in a hot plasma, thus fulfilling the magnetic flux conservation law. And it finally results in the present day intergalactic magnetic fields which could be correlated on $\sim 1 \mathrm{Mpc}$ scales. Note that an essential information on the processes that take place after the EWPT, obtained in the framework of magneto-hydrodynamics, is given in Refs. [17] and [28]. We will discuss the facts which have relevance to our problem in the last section.

Another possible scenario is based on the stochastic processes considered already by Hogan [29] in connection with the magnetic fields generated at first-order EWPT. A possible mechanism of field generation in that case was proposed by Vachaspati [27]. In the former paper, it was pointed out that magnetic fields correlated on large scales can be produced not only through causal processes but also by a stochastic random walk mechanism, if the magnetic lines gener- 
ated in some domain of space "forget" about their origin. The field strength developed on large scales by this process (due to "straightening" of entangled magnetic fields) can be estimated as $B_{N} \sim B / \sqrt{N}$, where $N$ counts the number of domains, with the field $B$ of a given size, crossed by a magnetic line. The correlation length $\lambda_{B}$ in this case can be much larger than the $R_{H}(T)$. It can be estimated as $\lambda_{B}(T) \sim N R_{H}(T)$. In the paper [29], it has been also noticed that this mechanism is not applicable to the early universe, the reason being because magnetic lines do not penetrate freely though the plasma. This is really the case, if the properties of the plasma as such are taken into account. However, this is not the case if spontaneous vacuum magnetization occurs. Actually, at a given temperature, each uncorrelated domain of space having a Hubble radius $R_{H}(T)$ is filled up with a constant magnetic field $B(T)$, described by the potential (2). Its orientation in both external and internal spaces is arbitrary. Hence, a stochastic behavior of the field lines and the appearance of magnetic fields having large correlation lengths $\lambda_{B}(T) \geq R_{H}(T)$ are expected. After the EWPT, these fields evolve as in the previous case.

Note that, in both scenarios, all the fields generated at the inflation epoch are washed out by the vacuum polarization and leave no remnants at present. The reheating stage becomes more important. In closing this section, we also notice that the long range nature of the Abelian spontaneously generated magnetic fields is ensured by their zero magnetic mass (see [15, 14]), what renders these fields unscreened, as is the case for usual $U(1)$ fields.

\section{Discussion and conclusions}

We here summarize our main results. The key point in the problem under investigation is the spontaneous vacuum magnetization process, which eliminates the magnetic flux conservation principle at high temperature. Vacuum polarization is responsible for the value of the field strength $B(T)$ at each temperature and serves as its source. In fact, it converts heat into an ordered coherent state, which was not taken into consideration in previous studies of the early Universe. We have also shown here that, at finite temperature and after symmetry breaking, a scalar field condensate suppresses the magnetization. Hence, it follows that the actual nature of the model extending the standard one is not that essential at sufficiently low temperatures, when the decoupling of the heavy gauge fields has occurred. From this one can conclude, in particular, that the vacuum polarization "washes out" the relics of the magnetic fields generated at very high temperature or at the inflation stage. These statements are new and come as an interesting surprise, as compared with the standard notions based on the ubiquitous scenario with magnetic flux conservation.

The present value of the intergalactic magnetic field strength has been here related with fields at high temperatures in the restored phase. Because of the 
zero magnetic mass for Abelian magnetic fields (as discovered recently [14, 15]), there is no problem for the generation of fields having large coherence scales. In our estimates, we have assumed that, basically, the field is of the order of the horizon scale, $\lambda_{B}(T) \sim R_{H(T)}$. This seems reasonable because, at a given temperature, the field $B(T)=$ const, generated due to vacuum polarization, occupies all space. Then, at the reheating stage (due to causality, present already at inflation), coherence on scales exceeding that of the horizon can be produced. Such large scale fields are not influenced by turbulent processes happening after the EWPT. They are frozen in the plasma and evolve according to the magnetic flux conservation law. In this scenario, a large scale domain structure is also permissible, what requires additional consideration.

Knowing the particular properties of the extended model, it is possible to estimate the field strengths at any temperature. This can be done for different schemes of spontaneous symmetry breaking (restoration) by taking into account the fact that, after the decoupling of some massive gauge fields, the corresponding magnetic fields are screened. Thus, the higher the temperature, the larger the number of strong long-range magnetic fields of different types that will be generated in the early Universe.

As we have found above, the field strengths at the EWPT temperature, estimated with account to the present-day value of the intergalactic magnetic field strength, $\sim 10^{-15} \mathrm{G}$, Eq. (20), or either directly from the vacuum magnetization in the standard model, Eq. (23), differ in six orders of magnitude. This huge deviation can be explained by the different scales of the fields considered. Let us check this possibility by using the second of the scenarios proposed in the previous section, for large scale field generation. Making use of the usual relation between the scale factor and the temperature,

$$
\frac{a\left(T_{e w}\right)}{a\left(T_{0}\right)}=\frac{T_{0}}{T_{e w}},
$$

taken at the EWPT epoch, and the present-day parameters, $T_{e w}=100 \mathrm{GeV}=$ $10^{11} \mathrm{eV}, T_{0}=2.3267 \cdot 10^{-4} \mathrm{eV}$. If one assumes that $\lambda_{B}(T) \sim a(T)$, then from (24), it follows that $\lambda_{B}\left(T_{0}\right)=6 \cdot 10^{-4}$ ps (see, for instance, [17]). On the other hand, if one takes $\lambda_{B}\left(T_{0}\right)=1 \mathrm{Mpc}$, the value $\xi_{B}\left(T_{e w}\right)=2.33 \cdot 10^{-15} \mathrm{Mpc}$ is obtained. At the same time, the horizon size is $a\left(T_{e w}\right)=1.27 \cdot 10^{-24} \mathrm{Mpc}$, thus, $\lambda_{B}\left(T_{e w}\right)>>$ $a\left(T_{e w}\right)$. Now, following an idea of Hogan [29, we relate the size of the correlated field with the random walk process. At $T_{e w}$, we have $\lambda_{B}\left(T_{e w}\right)=N a\left(T_{e w}\right)$, hence, we get roughly $\sqrt{N}=3 \cdot 10^{4}$, and for the field strength "straightened" on the $N$ domain scale, $B_{N} \sim \frac{B\left(T_{e w}\right)}{\sqrt{N}}[29]$. Therefore, accounting for the field strength value calculated for the standard model, Eq. (23), we obtain $B_{l s}\left(T_{e w}\right) \sim 3 \cdot 10^{15} \mathrm{G}$ (the subscript in $B_{l s}$ means "large scale"). This value is close the the value $B_{l s}\left(T_{e w}\right) \sim$ $2 \cdot 10^{14} \mathrm{G}$ estimated in Eq. (20). The remaining discrepancy can be explained in two ways. First, and obviously, as due to the roughness of our estimate. Second, 
and more radically, by the necessity of substituting the standard model with another one. The latter point will be discussed in more detail below.

Let us note a number of properties of the field under consideration, and compare them with the ones usually discussed in applications of magnetohydrodynamics to the early Universe. Here, we follow Refs. 17] and 28 which are close to our analysis. First, let us mention that the field energy density, $\rho_{B}=\frac{B^{2}}{2}$, is proportional to $g^{6} T^{4}$, what is much smaller than the radiation energy density, $\sim T^{4}$. Thus, the BBN condition (see [17]), $\rho_{B} / \rho_{\text {rad. }}<<1$, is fulfilled. Second, as numerical simulations show [17], the turbulent process in the early Universe with magnetic field included is slower then in the laboratory. Turbulence can include a large scale field at the level of the largest eddies. For large scale fields, the free decay stage is important. At this epoch, which is strongly dependent on the initial conditions [28], turbulence is significantly decreasing, and after this very brief stage the field is not affected by turbulence any more. It is just frozen in the plasma. As we have shown, the spontaneous vacuum magnetization is stopped when the first-order phase transition ends. Fields of this type cannot be influenced by turbulence and it is thus reasonable to believe that after the EWPT the field evolves according to Eq. (5). Note also that these fields are non-helical ones.

Our analysis has shown that, at the EWPT temperature, magnetic fields of the order $B\left(T_{e w}\right) \sim 10^{14} G$ did very likely exist. To estimate their field strengths at higher temperatures, one has to take into consideration a number of features proper to the standard model and its particular extension at play. First, we note that quarks possess both electric and color charges. Therefore, there is a mixing between the color and usual magnetic fields owing to the quark loops. Second, there are peculiarities related with the particular content of the extended models. For example, in the Two-Higgs-Doublet standard model the contribution $\sim(g B)^{3 / 2} T$ in Eq. (7) is exactly canceled by the corresponding term in Eq. (8)), because of the four charged scalar fields entering the model. They interact with gauge fields with the same coupling constant. However, in this model the doublets interact differently with fermions. This changes the effective couplings of the doublets with the gauge fields and results in non-complete cancelations. As a result, a suppression of the spontaneously created magnetic field is expected in this model. In principle, one should be able to explain, in this way, the discrepancy in the field strengths as discussed above. There can be other peculiarities which may influence the high temperature phase of the universe. They will require further investigation and thus we leave this issue for a future publication.

Acknowledgements. The authors are grateful to Michael Bordag for numerous discussions and a careful reading of the manuscript. VS was supported by the European Science Foundation CASIMIR Networking program. He also thanks the Group of Theoretical Physics and Cosmology, at the Institute for Space Sci- 
ence, UAB, Barcelona, for kind hospitality. EE's research was partly carried out while on leave at the Department of Physics and Astronomy, Dartmouth College, 6127 Wilder Laboratory, Hanover, NH 03755, USA. This work has been also partly supported by MICINN (Spain), projects FIS2006-02842 and FIS201015640, and Contract PR2011-0128, by the CPAN Consolider Ingenio Project, and by AGAUR (Generalitat de Catalunya), contract 2009SGR-994.

\section{References}

[1] S. Ando, A. Kusenko, Astrophys. J. Lett., 722, L39 (2010) arXiv:1005.1924 v2 [astro-ph.HE]].

[2] A. Neronov, E. Vovk, Science, 328, 73 (2010).

[3] W. Essey, S. Ando, A. Kusenko, Astropart. Phys. 35, 1351 (2011) arXiv:1012.5313.

[4] D. Grasso and H.R. Rubinstein, Phys. Rept. 348, 163 (2001).

[5] M. Giovannini, Int. J. Mod. Phys., D 13, 391 (2004).

[6] A. Kandus, K.E. Kunze, C.G. Tsagas, arXiv: 1007.3891 v2 [astro-ph. CO].

[7] A. O. Starinets, A. S. Vshivtsev and V. C. Zhukovsky, Phys. Lett. B 322, 403 (1994).

[8] K. Enqvist and P. Olesen, Phys. Lett. B 329, 195 (1994) arXiv:hep-ph/9402295.

[9] V. V. Skalozub, Int. J. Mod. Phys. A 11, 5643 (1996).

[10] V. Skalozub and M. Bordag, Nucl. Phys. B 576, 430 (2000). arXiv:hep-ph/9905302.

[11] V. I. Demchik and V. V. Skalozub, Phys. Atom. Nucl. 71, 180 (2008).

[12] G. K. Savvidy, Phys. Lett. B 71, 133 (1977).

[13] D. Ebert, V. C. Zhukovsky and A. S. Vshivtsev, Int. J. Mod. Phys. A 13, 1723 (1998).

[14] M. Bordag and V. Skalozub, Phys. Rev. D 75, 125003 (2007).

[15] S. Antropov, M. Bordag, V. Demchik and V. V. Skalozub, Int. J. Mod. Phys. A 26, 4831 (2011) [arXiv:hep-ph/1011.314].

[16] R. Banerjee and K. Jedamzik, Phys. Rev. D 70, 123003 (2004). 
[17] T. Kahniashvili, A.G. Tevzadze, B. Ratra, Astrophys. J. 726, v2, article 78 (2011) [astro-ph. CO].

[18] K. Goroku Progr.Theor.Phys., 68, No. 4, 1340 (1982).

[19] D. S. Gorbunov and V. A. Rubakov, Introduction to the Theory of the Early Universe: Cosmological Perturbations and Inflationary Theory (World Scien. Pub. Co., Singapore, 2011).

[20] M.D. Pollock, Int. J. Mod. Phys D 121289 (2003).

[21] V. Skalozub and V. Demchik, arXiv:hep-th/991207.

[22] M. Bordag, V. V. Skalozub. Phys. Rev. D 77, 105013 (2008).

[23] M.E. Carrington, Phys.Rev. D 45, 2933 (1992).

[24] V.I. Demchik and V.V. Skalozub, Eur. Phys. J. C 27, 601 (2003).

[25] V.I. Demchik and V.V. Skalozub, Eur. Phys. J. C 25, 291 (2002).

[26] R.P. Feynman, Nucl. Phys. B 188, 479 (1981).

[27] T. Vachaspati, Phys. Lett. B 265, 258 (1991).

[28] K. Subramanian and J. Barrow, Phys. Rev. D 58, 083502 (1998).

[29] C.J. Hogan, Phys. Re. Lett. 51, 1488 (1983). 